\title{
Four evolving concepts in molecular and clinical autonomic research
}

\author{
François M. Abboud ${ }^{1}$ \\ Received: 18 June 2021 / Accepted: 19 June 2021 / Published online: 30 June 2021 \\ c) Springer-Verlag GmbH Germany, part of Springer Nature 2021
}

Keywords Afferent $\cdot$ Sympathetic $\cdot$ Mechanosensors $\cdot$ PIEZO $\cdot$ ASIC $\cdot$ Neuroimmune $\cdot$ Microbiome $\cdot$ Microneurography

In reflecting on decades of autonomic research, I recall the 2009 Walter B. Cannon Memorial Award Lecture, which I entitled In Search of Autonomic Balance: The Good, The Bad and The Ugly [1]. The Good was the inhibition of excessive sympathetic nerve activity by baroreceptors and by cardiac and respiratory vagal afferents. The Bad was the loss of these inhibitory signals. The Ugly was the fact that the loss of the inhibitory signals enhances the excitatory signals (e.g., from chemoreceptors and somatic afferents). The discovery of the molecular determinants of afferent mechanical and chemical signaling, their paracrine activation, and their genetic regulation have revealed new mechanisms of cardiovascular autonomic dysfunction with potential therapeutic interventions. Namely, sophisticated optogenetic techniques allow activation of specific afferent autonomic nerves [16]; and novel surgical devices and approaches are used in clinical trials involving carotid sinus, glossopharyngeal, or vagus nerve stimulation, renal denervation, carotid body resection, and cardiac neuroablation.

In this invited commentary commemorating the 30th anniversary of Clinical Autonomic Research, I will highlight progress in clinical autonomic research by briefly reviewing four evolving concepts in the field. I ask for your indulgence, as I focus on experimental themes specifically pursued at the University of Iowa Cardiovascular Research Center. Consequently, other relevant autonomic concepts may not have been discussed here.

François M. Abboud

francois-abboud@uiowa.edu

1 Department of Internal Medicine, Edith King Pearson Chair of Cardiovascular Research, Abboud Cardiovascular Research Center, University of Iowa, 110 College of Medicine Administration Building (CMAB), Iowa City, IA 52242, USA

\section{First evolving concept: the neurosensory integration of human sympathetic activity and its clinical implications}

Hypertension, heart failure and obstructive sleep apnea are disorders with excessive sympathetic nerve activity as a major contributor to cardiovascular mortality. The convergence of multiple afferent signals, integrated centrally, defines the pattern of peripheral sympathetic activity. Additional examples of clinically significant sensory interactions abound. Thermal sensing by cutaneous afferents prevents reflex sympathetic vasoconstriction during hypotension to sustain preferentially heat dissipation, even though this could contribute to heat exhaustion and fainting [13]. Similarly, the reflex vasoconstrictor response to hypoxia is abrogated as a result of hyperventilation but is conversely enhanced, alarmingly, with the suppression of respiratory afferent activity during apnea. Using direct microneurography, we documented a dramatic and potentially fatal accentuation of sympathetic nerve activity in humans during obstructive sleep apnea [20]. (Fig. 1A).

Cardiac afferents are major regulators of the autonomic system supporting the concept that the heart is a sensory organ. Low-pressure mechanosensory vagal endings in the heart suppress sympathetic and enhance parasympathetic activity, regulating both blood volume and arterial pressure [2]. We established their role in neurocardiogenic syncope and syncope in patients with aortic stenosis. The distribution of these sensory endings in the posterior-inferior wall of the left ventricle explains the preponderance of bradycardia and hypotension in patients with inferior wall myocardial infarction in contrast to anterior wall infarction [22]. In patients with heart failure, treatment with cardiac glycosides, such as digitalis, restores the depressed sensitivity of these endings and thereby beneficially reduces excessive sympathetic nerve activity. Impairment of these endings is also seen with aging, hypertension, and diabetes mellitus. Although cardiac 
A
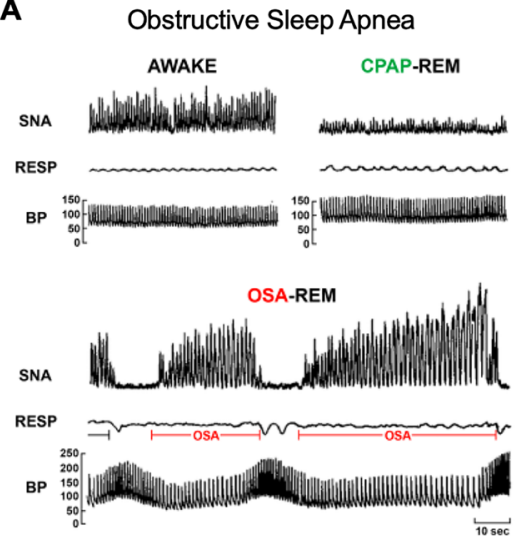

D Modulation of cytokine release from innate immune cells during TLR activation

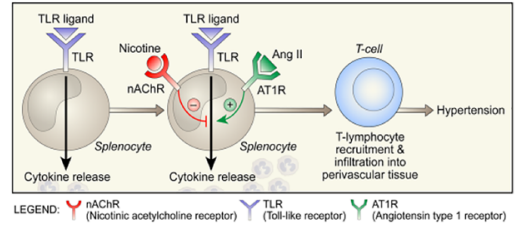

B
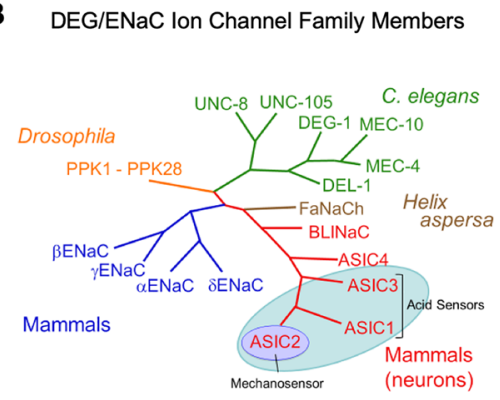

E

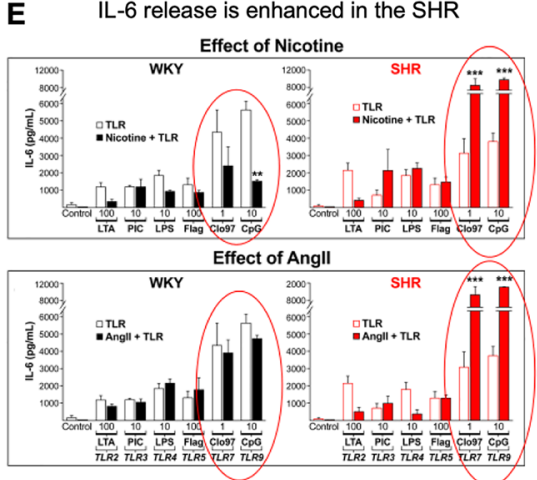

C Mechanically-Induced Depolarizations

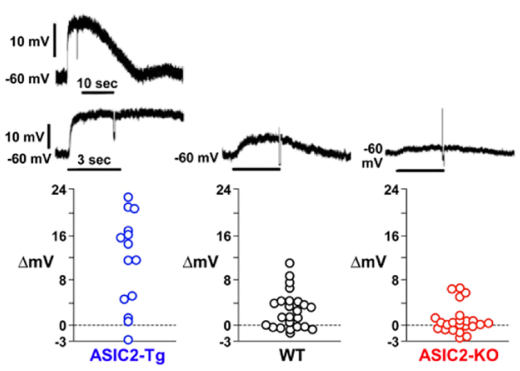

$\mathbf{F}$

Ang II response is abrogated in the Trif ${ }^{m u t}$ mice
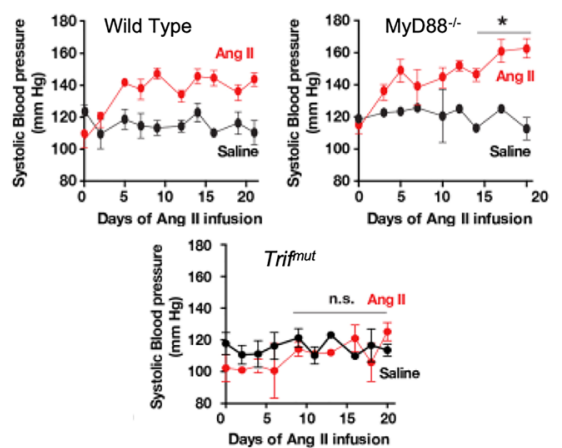

Fig. 1 Evolving concepts in molecular and clinical autonomic research. A Obstructive Sleep Apnea (OSA). Increased sympathetic nerve activity (SNA) in a patient with OSA is seen in the awake state and is enhanced alarmingly by episodes of OSA during the rapid eye movement (REM) sleep state. Positive airway pressure (CPAP) suppresses the increase in SNA during REM. Adapted from [20]. B Degenerin (DEG)/Epithelial Sodium Channel (ENaC) Family Members. Evolutionary conservation of mechanosensitive (MEC) and acid-sensing ion channels (ASICs). Adapted from [1]. C Mechanically induced Depolarization of aortic baroreceptor neurons is ASIC2 dependent. Adapted from [15]. D Cytokine release from innate immune cells in response to Toll-like receptor (TLR) ligands is regulated by nicotine and angiotensin II (Ang II) receptors. Adapted from [3]. E Response of splenocytes to Toll-like receptor (TLR) activation is suppressed by nicotine in normotensive rats (WKY) and enhanced by both nicotine and angiotensin II (Ang II) in spontaneously hypertensive rats (SHR). Adapted from [3]. F Pressor responses to angiotensin II (Ang II) are dependent on two adaptor proteins that mediate Toll-like receptor (TLR) activation of innate immune responses. MyD88 suppresses and TRIF mediates the pressor response and the pro-inflammatory gene expression. Adapted from [19]

CNS nuclei to activate the sympathetic nervous system to white and brown adipose tissues in a positive feedback reflex termed adipose afferent reflex. This reflex provides a neural control of energy balance. When lipolysis is high in white adipose tissue, the thermogenic machinery in brown adipose tissue is engaged [9].

\section{Second evolving concept: the molecular determinants of afferent sensory signals}

In the past three decades, our work led to: i) the discovery that, in addition to the classical baro- and chemoreceptors, rheoreceptors, specialized receptors that respond to changes in blood flow, contribute to carotid sinus nerve activity [10]; and ii) that endocrine, paracrine-endothelial, and autocrine ionic and oxidative processes are powerful modulators of neurosensory activity. For example, decreased synthesis of endogenous prostanoids as well as 
increased reactive oxygen species suppresses baroreceptor afferent signals in hypertension and atherosclerosis. We demonstrated that the resetting of the baroreceptors is predominantly neuronal rather than vascular, identified mechanosensitive ion channels in isolated patch-clamped baroreceptor neurons, and found that pulsatility of the arterial pressure signal prevents baroreceptor afferent adaptation and sustains reflex sympathoinhibition. We also reported that the mechanoelectrical transduction in baroreceptor neurons is mediated by a new class of gadolinium-sensitive stretch-activated channels. These mechanosensitive channels, initially identified in differentiated touch receptor neurons of C. elegans [5], are members of an invertebrate subfamily of the Degnerin (DEG)/ epithelial sodium channel (ENaC) family (Fig. 1B). In mammals, a subfamily of the DEG/ENaC family includes the Acid Sensing Ion Channels (ASIC), which are expressed in human peripheral nerve and brain [25]. ASIC have a broad range of sensitivities not just to acid $\mathrm{pH}$ and protons, but also to mechanical deformation, temperature change, and nociception. The ASIC subunits 1, 2, and 3 in different heteromeric combinations form various channels in different nerve terminals and define the nature of the sensory signals. Using gene deletion and overexpression strategies, we defined a critically important role of these and other sensory molecules [8] in maintaining autonomic balance. For instance, in aortic baroreceptor neurons, ASIC 2 is an important determinant of mechanically induced depolarization [15] (Fig. 1C). And the ASIC 2 knock-out mouse develops neurogenic hypertension. We also reported that blockade of potassium channels enhances the generation of action potentials in nodose ganglion neurons [6], and that activation of the sodium-potassium ATPase causes baroreceptor resetting during acute hypertension [12]. These ionic mechanisms play a role in the impaired baroreceptor activity in hypertension.

Patch-clamping glomus cells of the carotid bodies uncovered ion channels that are hypersensitive to hypoxia and low $\mathrm{pH}$ in these peripheral chemoreceptors. Specifically, non-voltage gated acid-sensing ion channels (ASIC 1 and ASIC 3) and two-pore domain transient acid-sensing potassium channels (TASK) are overexpressed and linked to the exaggerated chemoreceptor sensitivity in the spontaneously hypertensive rat compared to normotensive rats [21]. In skeletal muscle afferents, ASIC heteromers contribute significantly to the exercise pressor reflex, and, in cardiac afferents of the dorsal root ganglia, ASICs contribute to nociception and the detrimental sympathetic activation during cardiac ischemia and heart failure. The translational importance of activation of ASIC 2 in baroreceptors is a beneficial suppression of the enhanced sympathetic excitatory reflexes of chemoreceptors and skeletal muscle afferents, as well as the ASIC1a-mediated excitation of
CNS neurons during emotional states of fear, threat, or panic.

Piezo1 and Piezo2 are mechanically activated ion channels that play crucial roles in several mechanotransduction processes. In collaboration with Drs. Liberles and Patapoutian, we recently reported that Piezo ion channels are expressed in sensory neurons that contribute to the baroreflex. Conditional double knock-out of the PIEZO1 and PIEZO2 genes in mice causes baroreflex failure severely disrupting control of blood pressure and heart rate [26]. Patients with PIEZO2 mutations have severely decreased proprioception [7]. Combined loss of function of Piezo1 and Piezo2 presumably causes baroreflex failure in humans, although these patients are yet to be identified.

\section{Third evolving concept: the autonomic neuro-immune synapse}

Autonomic regulation of the immune system has a major influence on cardiovascular disease and clinical outcomes. Increases in the sympathetic and the renin-angiotensin system (RAS) activities and decreases in parasympathetic cholinergic activity are vital in acute circulatory adjustments to hemodynamic, hypotensive stresses. When sustained and prolonged, these autonomic changes have nefarious consequences [1]. The destructive tissue damage is not the result of the hemodynamic influence per se but rather a powerful autonomic pro-inflammatory modulation of a genetically vulnerable immune system [18] (Fig. 1D and 1E). Activation of the sympathetic and the renin-angiotensin systems is pro-inflammatory, whereas parasympathetic activation is anti-inflammatory and protective. The anti-inflammatory effect is mediated by the $\alpha 7$ nicotinic cholinergic receptor $(\alpha 7 \mathrm{nAChR})$ on innate immune cells [23]. Deletion of this receptor enhances end-organ damage in different models of hypertension, whereas its pharmacologic stimulation is protective.

This can be studied in the spontaneously hypertensive rat (SHR), a clinically relevant genetic animal model of hypertension. During a pre-hypertensive period of up to 6 weeks after birth, when the SHR newborn is still normotensive, significant autonomic, metabolic, and immunological abnormalities occur. For example, SHR splenocytes exposed to $\mathrm{CpG}$, a ligand of Toll-like receptor 9 (TLR9) activation, release large quantities of pro-inflammatory IL- 6 and IL- $1 \beta$ when co-stimulated with nicotine. This proinflammatory reaction is not observed in the normotensive control Wistar-Kyoto rat, in which nicotine suppresses the pro-inflammatory response to CpG. Harwani and colleagues, at the University of Iowa, propose that nicotinic cholinergic receptors mediate either anti- or pro-inflammatory responses depending on the activation of selective nicotinic receptors 
that may be differentially expressed in innate immune cells of SHR vs. normotensive control rats [11] (Fig. 1E). Abnormal innate immune responses may also be defined by the TLRs that are activated, their ligands, and their adaptor proteins. For example, we recently confirmed that angiotensin II (Ang II) responses in mice depend on two major adaptor proteins: MyD88 (myeloid protein 88 ) and TRIF (TIR-domain-containing adaptor-inducing interferon- $\beta$ ) [19]. A non-functional TRIF abrogates Ang II-induced hypertension, cardiac hypertrophy, and pro-inflammatory gene expression, whereas deletion of MyD88 enhances these responses (Fig. 1F). This indicates that MyD88 is a protective negative regulator of the inflammatory responses to Ang II.

\section{Fourth evolving concept: the regulation of intestinal vagal afferents and their microbiome interactions}

The satiety effects and metabolic actions of cholecystokinin (CCK) have been viewed as potential therapeutic targets for obesity. We identified a $\mathrm{Ca}^{2+}$-activated chloride $\left(\mathrm{Cl}^{-}\right)$current $(\mathrm{CaCC})$ that is evoked by $\mathrm{CCK}$ in intestinal vagal afferents of nodose neurons [24]. The CaCC subunit Anoctamin 2 (Ano2/TMEM16B) is the dominant contributor to this current. Reduced expression of TMEM16B in the heterozygote knock-out of the channel in sensory neurons results in obese rats with loss of CCK sensitivity in intestinal nodose neurons, loss of CCK-induced satiety, and decreased energy expenditure. Our findings reveal that Ano2/TMEM16B expressed in sensory neurons selectively promotes a reflex increase in sympathetic nerve activity to brown adipose tissue and corresponding thermogenesis [24].

Chemical signals from the gut microbiota activate vagal afferent terminals beneath the gut epithelium and affect food intake and metabolism. They may trigger inflammatory responses in vagal afferent neurons with cell bodies located in nodose ganglia, causing autonomic dysfunction [4]. In addition, nodose ganglia microglial activation by microbiota dysbiosis may suppress vagal afferent signals to the CNS. We found remarkable differences in neonatal gut microbiota between SHR and normotensive control rats that do not persist into adulthood and yet may have a lasting effect on the immune response and the development of hypertension [16]. More importantly, cross-fostering of SHR with normotensive control rats changes their neonatal microbiota to reflect that of the normotensive control rats and favorably reduces the inflammatory response and hypertension. Although cross-fostering also induces changes in the microbiota of normotensive control rats toward neonatal SHR microbiota, these changes are not sufficient to cause hypertension [17]. We suspect that the likely source of the neonatal diversity could be the mother's milk, the genetic predisposition of the pups in supporting the growth of specific early gut bacteria, or both. Therefore, cross-fostering is a robust method of altering the neonatal gut microbiota with distinct and measurable long-term beneficial outcomes, with potential implications in sympathetic-mediated disorders such as hypertension.

\section{Funding sources}

NHLBI Grants HL-14388 and HL-0721.

\section{Declarations}

Conflict of interest None.

\section{References}

1. Abboud FM (2010) The Walter B. Cannon Memorial Award Lecture, 2009. Physiology in perspective: The wisdom of the body. In search of autonomic balance: the good, the bad, and the ugly. Am J Physiol Regul Integr Comp Physiol 298:R1449-1467

2. Abboud FM, Eckberg DL, Johannsen UJ, Mark AL (1979) Carotid and cardiopulmonary baroreceptor control of splanchnic and forearm vascular resistance during venous pooling in man. J Physiol 286:173-184

3. Abboud FM, Singh MV (2017) Autonomic regulation of the immune system in cardiovascular diseases. Adv Physiol Educ 41:578-593

4. Cawthon CR, de La Serre CB (2018) Gut bacteria interaction with vagal afferents. Brain Res 1693:134-139

5. Chalfie M, Au M (1989) Genetic control of differentiation of the Caenorhabditis elegans touch receptor neurons. Science 243:1027-1033

6. Chapleau MW, Lu J, Hajduczok G, Abboud FM (1993) Mechanism of baroreceptor adaptation in dogs: attenuation of adaptation by the $\mathrm{K}+$ channel blocker 4 -aminopyridine. J Physiol 462:291-306

7. Chesler AT, Szczot M, Bharucha-Goebel D, Ceko M, Donkervoort S, Laubacher C, Hayes LH, Alter K, Zampieri C, Stanley C, Innes AM, Mah JK, Grosmann CM, Bradley N, Nguyen D, Foley AR, Le Pichon CE, Bonnemann CG (2016) The Role of PIEZO2 in Human Mechanosensation. N Engl J Med 375:1355-1364

8. Drummond HA, Price MP, Welsh MJ, Abboud FM (1998) A molecular component of the arterial baroreceptor mechanotransducer. Neuron 21:1435-1441

9. Garretson JT, Szymanski LA, Schwartz GJ, Xue B, Ryu V, Bartness TJ (2016) Lipolysis sensation by white fat afferent nerves triggers brown fat thermogenesis. Mol Metab 5:626-634

10. Hajduczok G, Chapleau MW, Abboud FM (1988) Rheoreceptors in the carotid sinus of dog. Proc Natl Acad Sci U S A 85:7399-7403

11. Harwani SC, Ratcliff J, Sutterwala FS, Ballas ZK, Meyerholz DK, Chapleau MW, Abboud FM (2016) Nicotine Mediates CD161a+ Renal Macrophage Infiltration and Premature Hypertension in the Spontaneously Hypertensive Rat. Circ Res 119:1101-1115

12. Heesch CM, Abboud FM, Thames MD (1984) Acute resetting of carotid sinus baroreceptors. II. Possible involvement of electrogenic Na+ pump. Am J Physiol 247:H833-839 
13. Heistad DD, Abboud FM, Mark AL, Schmid PG (1973) Interaction of thermal and baroreceptor reflexes in man. J Appl Physiol 35:581-586

14. Heistad DD, Abboud FM, Mark AL, Schmid PG (1974) Interaction of baroreceptor and chemoreceptor reflexes. Modulation of the chemoreceptor reflex by changes in baroreceptor activity. $\mathrm{J}$ Clin Invest 53:1226-1236

15. Lu Y, Ma X, Sabharwal R, Snitsarev V, Morgan D, Rahmouni K, Drummond HA, Whiteis CA, Costa V, Price M, Benson C, Welsh MJ, Chapleau MW, Abboud FM (2009) The ion channel ASIC2 is required for baroreceptor and autonomic control of the circulation. Neuron 64:885-897

16. Rajendran PS, Challis RC, Fowlkes CC, Hanna P, Tompkins JD, Jordan MC, Hiyari S, Gabris-Weber BA, Greenbaum A, Chan KY, Deverman BE, Munzberg H, Ardell JL, Salama G, Gradinaru V, Shivkumar K (2019) Identification of peripheral neural circuits that regulate heart rate using optogenetic and viral vector strategies. Nat Commun 10:1944

17. Singh MV, Cicha MZ, Ericsson A, Chapleau MW, Abboud FM (2019) Influence of Early Postnatal Gut Microbiota on Immune System in SHR Hypertension. FASEB J 33:692.616-692.616

18. Singh MV, Cicha MZ, Kumar S, Meyerholz DK, Irani K, Chapleau MW, Abboud FM (2017) Abnormal CD161(+) immune cells and retinoic acid receptor-related orphan receptor gammat-mediate enhanced IL-17F expression in the setting of genetic hypertension. J Allergy Clin Immunol 140:809-821 e803

19. Singh MV, Cicha MZ, Meyerholz DK, Chapleau MW, Abboud FM (2015) Dual Activation of TRIF and MyD88 Adaptor Proteins by Angiotensin II Evokes Opposing Effects on Pressure, Cardiac Hypertrophy, and Inflammatory Gene Expression. Hypertension 66:647-656

20. Somers VK, Dyken ME, Clary MP, Abboud FM (1995) Sympathetic neural mechanisms in obstructive sleep apnea. J Clin Invest 96:1897-1904

21. Tan ZY, Lu Y, Whiteis CA, Simms AE, Paton JF, Chapleau MW, Abboud FM (2010) Chemoreceptor hypersensitivity, sympathetic excitation, and overexpression of ASIC and TASK channels before the onset of hypertension in SHR. Circ Res 106:536-545

22. Thames MD, Klopfenstein HS, Abboud FM, Mark AL, Walker JL (1978) Preferential distribution of inhibitory cardiac receptors with vagal afferents to the inferoposterior wall of the left ventricle activated during coronary occlusion in the dog. Circ Res 43:512-519

23. Tracey KJ (2002) The inflammatory reflex. Nature 420:853-859

24. Wang R, Lu Y, Cicha MZ, Singh MV, Benson CJ, Madden CJ, Chapleau MW, Abboud FM (2019) TMEM16B determines cholecystokinin sensitivity of intestinal vagal afferents of nodose neurons. JCI Insight 4(5): 122058

25. Wemmie JA, Price MP, Welsh MJ (2006) Acid-sensing ion channels: advances, questions and therapeutic opportunities. Trends Neurosci 29:578-586

26. Zeng WZ, Marshall KL, Min S, Daou I, Chapleau MW, Abboud FM, Liberles SD, Patapoutian A (2018) PIEZOs mediate neuronal sensing of blood pressure and the baroreceptor reflex. Science 362:464-467 